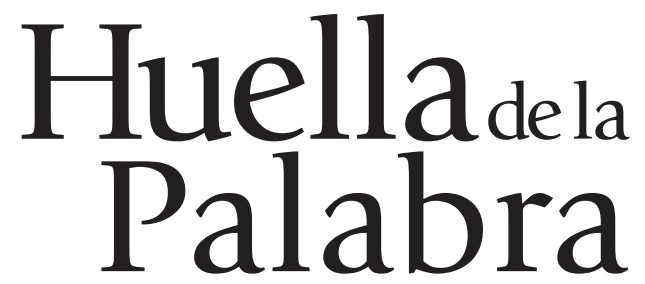

Revista de Investigación Educativa

Universidad La Salle Pachuca

lahuelladelapalabra@lasallep.edu.mx

Teléfono: 01(771) 7170213 ext. 1406

Fax: $01(771) 7170309$

ISSN: En trámite

México

Erika López López

Duelo en pacientes con diagnóstico de diabetes mellitus y servicios de salud culturalmente competentes: propuestas de abordaje desde la psicología

Huella de la Palabra, año 2016, número 10

Universidad La Salle Pachuca

pp. $71-82$ 



\section{Duelo en pacientes con diagnóstico de diabetes mellitus y servicios de salud culturalmente competentes: propuestas de abordaje desde la psicología \\ Erika López López ${ }^{1}$

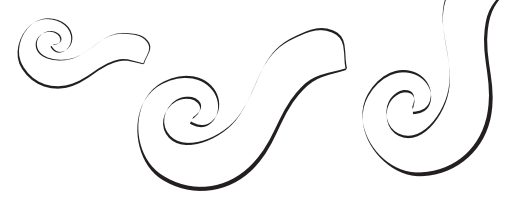

Resumen

La Diabetes Mellitus es una enfermedad crónica que representa un reto para el paciente, su familia y el sistema de salud. A pesar de las acciones llevadas a cabo, no se ha podido contrarrestar esta epidemia. En este documento se propone integrar fundamentos desde una perspectiva psicológica y tanatológica para abordar al paciente con DM y preparar a los Servicios de Salud desde la competencia cultural con el fin de capacitar al personal de salud con herramientas y promover ambientes respetuosos, de servicio y de atención hacia los pacientes.

\section{Introducción}

La DM es un trastorno metabólico que afecta tanto a órganos como a tejidos, se caracteriza por un aumento de los niveles de glucosa en la sangre y se clasifica en DM tipo 1, DM tipo 2 y diabetes gestacional (ADA, 2011; IEC,2009). De acuerdo con el Atlas de la Diabetes de la Federación Internacional de Diabetes, en 2015 había 415 millones de adultos con DM y se espera que en 2040 sean 642 millones (FIC, 2015).

En México, información de la Dirección General de Epidemiología se la Secretaría de Salud registró 29058 casos de pacientes con DM (DGE, 2015). De acuerdo al reporte mensual de agosto de 2016, en Hidalgo se reportaron 7 221 casos, de los cuales el 39.1\% corresponden a la Secretaría de Salud de Hidalgo, 37.4\% al Instituto de Seguridad y Servicios Sociales de los Trabajadores del Estado (ISSSTE), 17.4\% Instituto Mexicano del Seguro Social (IMSS) y el $4.8 \%$ al IMSS-Prospera, el resto se diluye en otras instituciones (DGE, 2016). Esto quiere decir que existen 2.5 personas con DM por cada mil habitantes en Hidalgo ${ }^{2}$. Lo anterior, permite visualizar el panorama general de esta enfermedad y permite comprender el siguiente reto para el paciente, su familia y el sistema de salud: el tratamiento.

El tratamiento del paciente con DM es un fenómeno que tiene diversas implicaciones psicológicas, sociales, clínicas y económicas. Afortunadamente, si se interviene de manera oportuna, la evolución natural de la DM puede modificarse y prevenir serias complicaciones que podrían ser fatales. Por ello, el abordaje del profesional en psicología implica conocer la dinámica de la enfermedad, su tratamiento, la rehabilitación psicosocial y la prevención de complicaciones. La educación para la salud en las personas con DM o educación en DM ha adquirido enorme relevancia hasta el punto en que se le considera un factor clave del tratamiento (OMS, 2000). La adherencia terapéutica es el fenómeno en el que el paciente adquiere conductas asertivas para iniciar el tratamiento: continúa con el tratamiento prescrito, cumple con las indicaciones respecto a las dosis, el ejercicio y el régimen

1 Licenciada en Psicología por la Universidad Autónoma del Estado de Hidalgo, Maestra en Ciencias de la Salud por el Instituto Nacional de Salud Pública y actualmente es doctorante en Estudios de Población por el Colegio de México.

2 Cifras de INEGI reportan que en 2015 vivían 2,858,359 habitantes en Hidalgo. 


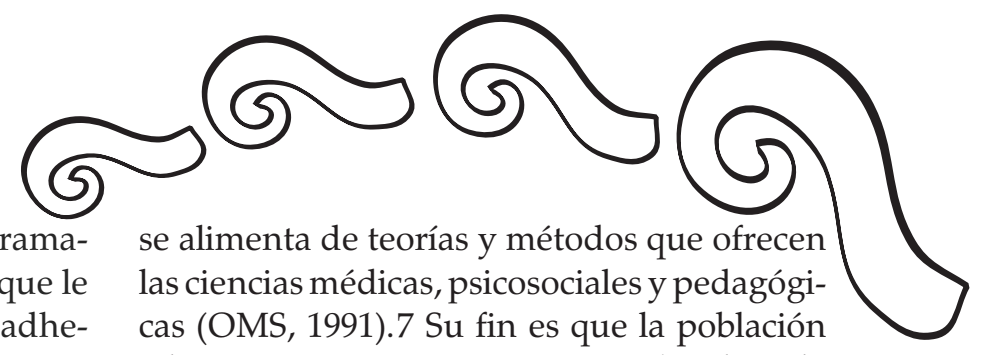

alimenticio; acude a las consultas programadas y adopta los nuevos estilos de vida que le permitan lograr las metas clínicas. La adherencia terapéutica se considera efectiva cuando el paciente comprende la importancia de un adecuado control glucémico, qué necesita hacer para conseguirlo e identifica cuáles son las estrategias adecuadas para resolver los problemas que se le presenten.

La Organización Mundial de la Salud (OMS) propone la educación en DM como elemento fundamental en el tratamiento y la única solución eficaz para el control de la enfermedad y la prevención de sus complicaciones (OMS, 1991). Sin embargo, dentro de los servicios de salud, ¿Quiénes son los responsables de llevarlo a cabo? ¿El área de medicina, enfermería, trabajo social o de psicología? ¿Quiénes son los que se forman para empoderar al paciente frente a una enfermedad? ¿Los profesionales de la salud comprenden los procesos psicológicos y mecanismos de defensa cuando se presenta un diagnóstico como DM? ¿Quiénes son los profesionales que son formados para comprender las dinámicas familiares frente a una pérdida? ¿Quiénes son los que estudian los procesos cognoscitivos $\mathrm{y}$ culturales implicados en el apego al tratamiento de una enfermedad crónica?

Evidentemente, existe un sesgo por la formación psicológica; sin embargo, proponer que el profesional de la salud mental esté empoderado para actuar en este ámbito, es debido a que durante su formación adquiere herramientas que le permiten comprender las dinámicas intersubjetivas que tienen alcances en la clínica y que impactan en materia de salud pública. La evidencia de los beneficios de programas educativos en DM permite observar que la educación no mejora únicamente la calidad de vida, los conocimientos y la toma de decisiones, sino que además reduce el costo sanitario y social de la enfermedad (Puente y cols., 1999; DGE, 1994).

La educación en DM es un proceso social que adopte y mantenga prácticas, estilos de vida saludables y propicie cambios socioculturales (Salleras, 2005). Una vez que el paciente cuenta con los conocimientos y destrezas necesarias, puede afrontar asertivamente exigencias del tratamiento, así como promover en él las motivaciones y los sentimientos de seguridad y responsabilidad para cuidar diariamente de su control, sin afectar su autoestima y bienestar general (García y Suárez, 2007).

En la Secretaría de Salud de México, los grupos de ayuda mutua (GAM) han sido una estrategia que ha permitido brindar información sobre estaa enfermedad a quienes asisten. Dicha estrategia forma parte del Programa de Salud del Adulto y el Anciano del Centro Nacional de Vigilancia Epidemiológica y Control de Enfermedades (CENAVECE). Ésta tiene el objetivo de estimular a los integrantes a adoptar estilos de vida saludables, ya que la educación, la actividad física y la alimentación del paciente son elementos clave e indispensables del tratamiento (SSA, 1998).

Un objetivo fundamental de los GAM es crear redes de apoyo social y empoderar a personas con DM en el autocuidado, así como facilitar y promover su capacitación. La propuesta inicial contempló su incorporación en unidades de atención del Sistema Nacional de Salud, en centros de trabajo, escuelas y otras organizaciones de la sociedad civil (SSA, 2010). Actualmente los GAM se han formado principalmente en las unidades de salud del primer nivel de atención (Lara y cols., 2004). Las sesiones educativas permiten el intercambio de experiencias en un clima de confianza, pero con información sencilla y muy concreta que guían a los integrantes (SSA, 2011). Para complementar las sesiones, un profesional de la salud capacitado está en constante supervisión (SSA, 1998).

A pesar de los esfuerzos en Hidalgo, el control 


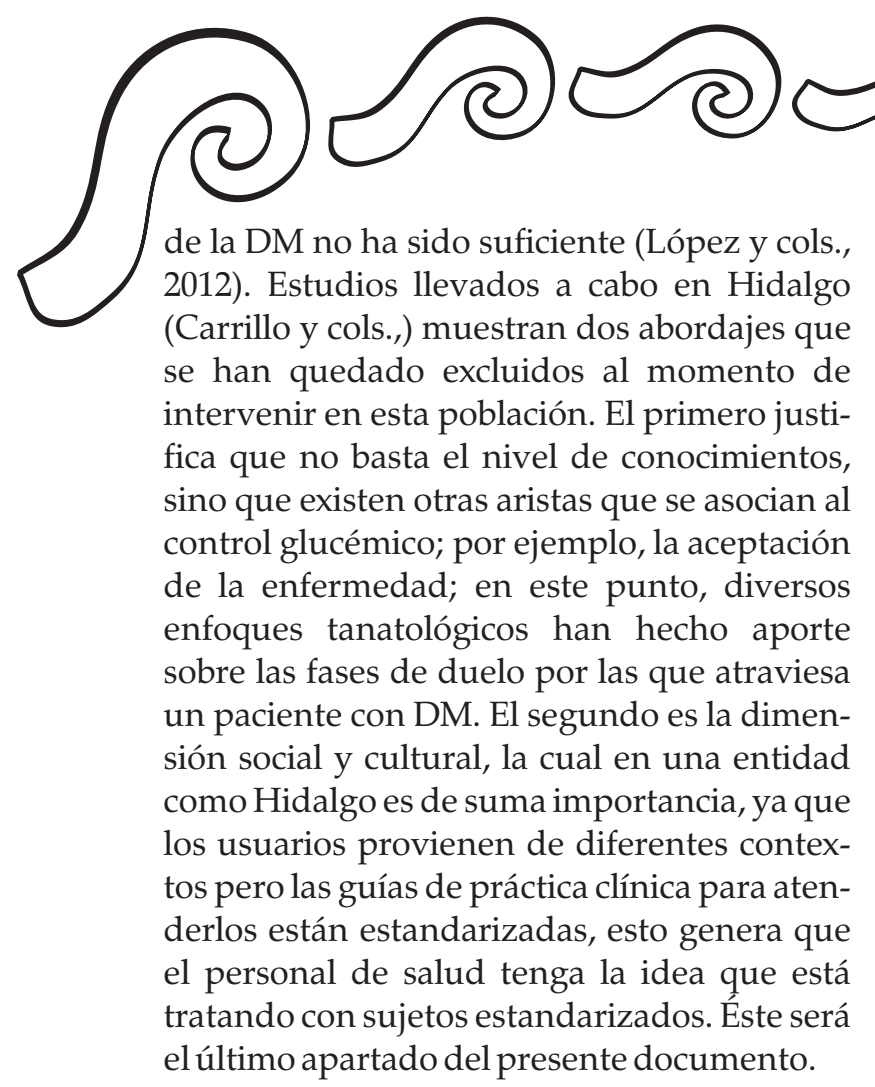

El contexto sociocultural del paciente en el proceso salud-enfermedad

Uno de los estudios que muestra el impacto sociocultural es A 'beautiful death': Mortality, death, and holidays in a Mexican municipality (Wilches y cols., 2012), el cual fue un diseño cuantitativo y cualitativo que expuso cómo la muerte se presenta durante días festivos. La muerte bonita se relaciona con la expectativa de una buena muerte y la naturaleza muy especial de la muerte durante unas vacaciones, debido a la participación de las entidades religiosas, como Dios, la Virgen María, y / o un santo, en el momento de la muerte. Los resultados muestran los efectos importantes de las vacaciones, la cultura y las creencias religiosas en los patrones de mortalidad dentro de un contexto mexicano, y ha contribuido a una mejor comprensión de las relaciones entre la mortalidad, la naturaleza de la muerte, y los días festivos.

El paciente con DM introyecta elementos psicológicos del proceso salud-enfermedadatención-muerte, esto le permite al personal de salud identificar cuáles son las representa- ciones sociales que el paciente tiene sobre su enfermedad. La familia, como la primera institución social y en la que el individuo se desarrolla, genera un espacio particular donde transmite las pautas y creencias de la cultura dominante (Moxley y cols., 2004). De acuerdo con Jodelet, estas representaciones sociales se entienden como "saberes del sentido común que orientan la acción, la comunicación y la comprensión del entorno social, material o ideal" (Jodelet, 1993), lo cual es determinante para lograr la aceptación de la enfermedad, ya que la mayoría de las veces la representación social de la DM en los profesionales de la salud y el paciente se presentan como opuestas. En este sentido, comprender las creencias, sistemas de pensamiento y prácticas cotidianas que los pacientes llevan a cabo en pro de restaurar su condición de salud, puede ser de gran utilidad para los profesionales de la salud (Torres y cols., 2005).

\section{Abordaje tanatológico del paciente con DM}

Una vez que el médico da el diagnóstico de $\mathrm{DM}$, el paciente entra en un proceso de duelo, perdió el imaginario de la salud, su concepto de estilo de vida, su autoconcepto y su autoimagen. Esta experiencia es única y determinante para muchos de los pacientes, pues la manera en que es transmitida la noticia, influye en la respuesta del paciente.

En su famoso libro Sobre la muerte y los moribundos, Elizabeth Kübler Ross (1969) describe las cinco fases que identificó frente a una pérdida, en las cuales el profesional de la salud puede intervenir estratégicamente y promover el apego al tratamiento de manera efectiva. Estas fases son las siguientes:

1. Negación. Es un mecanismo de defensa que también ha sido descrito por el ámbito psicoanalista. Esta etapa fue definida como la imposibilidad de aceptar y reconocer como real el diagnóstico de DM y se consideran justificaciones racionales como errores médicos, de laboratorio o algunos relacionados con 


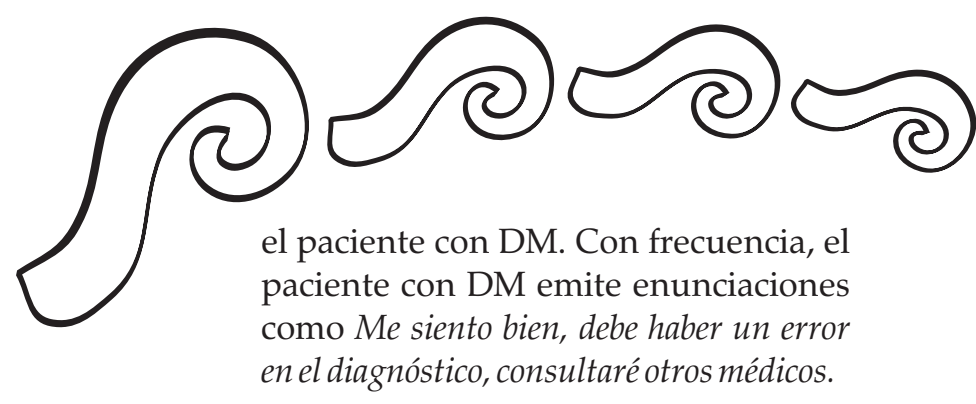

2. Ira. En esta etapa el paciente experimenta emociones como rabia y coraje por haber perdido la salud, reconocer que esta enfermedad no tiene cura y el tratamiento es crónico. Asimismo, experimenta envidia, resentimiento y hostilidad hacia individuos que lo rodean y que representan salud o vitalidad, así que estos sentimientos los proyecta hacia personal sanitario, familiares, amistades, divinidades en quienes cree (Dios, Santos, etc.) o elementos simbólicos (castigos divinos). También puede dirigirse esta ira hacia sí mismo y tiende a culparse por haber provocado la enfermedad.

3. Negociación o pacto. Durante esta etapa se desarrollan mecanismos psicológicos para afrontar la culpa a través de acuerdos para alcanzar las metas clínicas con personas cercanas como familiares o personal de salud. Asimismo, quienes tienen un sistema de creencias espiritual y religioso, los acuerdos los hacen con las divinidades de carácter superior, como Dios, Jesús o seres de otras religiones. Las conductas autodestructivas se muestran latentes y emerge una actitud de apego al tratamiento, ya que la esperanza genera en el individuo el desarrollo de mecanismos simbólicos sobre el aplazamiento de su muerte.

4. Depresión. Sin duda, esta etapa es de mayor vulnerabilidad, ya que los mecanismos inmunológicos juegan un papel muy importante sobre el control glucémico. Emergen sentimientos de vacío, pierde el sentido a la vida y tiene ideas de la inminente muerte y tienden a ser herméticos. Es muy importante vigilar esta fase, ya que con frecuencia, el paciente abandona el tratamiento y no acude a las citas médicas.

5. Aceptación. En esta última etapa existe un reconocimiento de la enfermedad, su tratamiento, la prevención de las complicaciones y un desenvolvimiento asertivo con quienes lo rodean. Asume una actitud responsable frente a sí mismo y emergen nuevos significados a la situación que se vive. Es importante señalar que no es una resignación ante la enfermedad o alcanzar un estado de felicidad, sino un proceso cognoscitivo de adoptar la enfermedad como una extensión de sí mismo y no un impedimento para tener vitalidad y sentido a su vida. Justo en esta etapa, el logro de la adherencia terapéutica ocurre de manera exitosa.

Este proceso de duelo no es lineal y puede haber interacciones entre las fases o retrocesos. Cada paciente lo enfrenta con sus propias herramientas, algunos de ellos solicitan el apoyo de expertos de la salud mental con la finalidad de lograr la aceptación. De tal manera que puedan sentirse libres de expresar sus emociones y contar con un acompañamiento profesional. Justo en este momento, los Servicios de Salud toman un papel importante, ya que uno de los deberes del Estado es proteger la salud de la población. Pero, ¿qué capacidad tienen para abordar estos fenómenos complejos que viven miles de ciudadanos a quienes se les diagnostica alguna enfermedad crónica? ¿Qué herramientas tienen para atender e identificar a las diferentes fases en las que se encuentra cada uno de los pacientes? ¿Cuentan con las herramientas adecuadas para transmitir el diagnóstico a una persona analfabeta y a una con educación a nivel superior? Justo hace unos años, surge la propuesta de competencia cultural en los Servicios de Salud. 


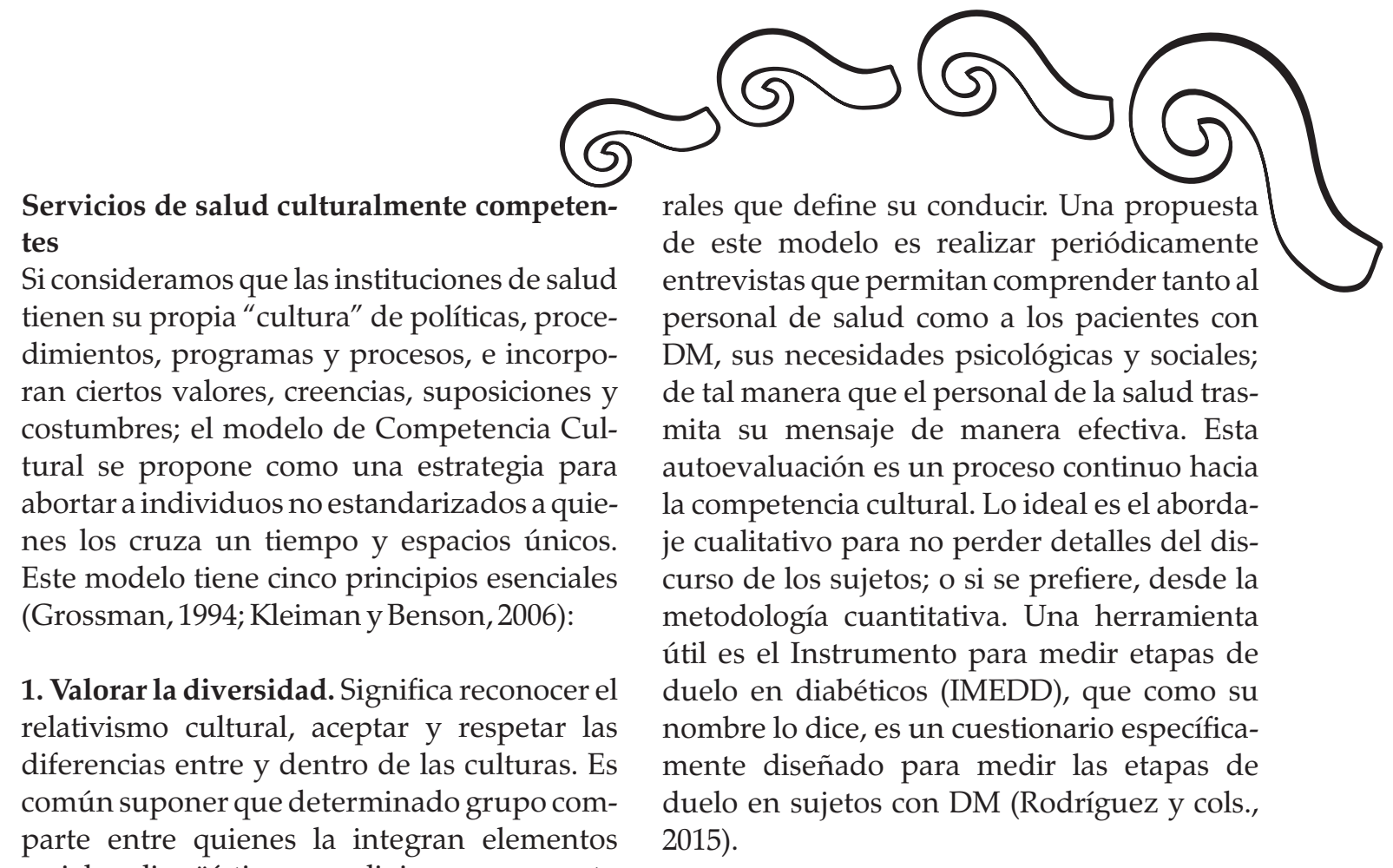
raciales, lingüísticos y religiosos, pero esto puede no ser cierto o el individuo los niega. Por ejemplo, la desindianización que sufrieron los mayas, quienes se divorciaron parcialmente de los significados de la indianidad, entre ellas algunas prácticas y costumbres indígenas (Lizama y Loret, 2010). La propuesta de este principio, ocurre cuando un grupo comparte experiencias históricas y geográficas, pero sus individuos pueden compartir únicamente su apariencia, idioma o creencias espirituales. El peligro emerge cuando suponemos un actuar intrínseco por pertenecer a determinados grupos y emitir conclusiones equivocadas. La etnicidad, la educación, la localidad y el estatus socioeconómico en el paciente con DM pueden influir determinantemente en la aceptación de la enfermedad. Por ejemplo, el consumo de ciertos menjurjes que la medicina tradicional mexicana promueve, o la demanda excesiva de los productos milagro versus la confianza en la medicina alópata. En este apartado podemos observar que aunque existan dos pacientes con las mismas características, es posible que estén en fases del duelo diferentes, lo cual implica un abordaje específico para cada uno.

2. Conducir una autoevaluación cultural. Cada organización tiene características cultu-

3. Comprender la dinámica de las diferencias. Existen factores que afectan las dinámicas culturales, como pueden ser experiencias históricas. Por ejemplo, personas que han sufrido discriminación y trato injusto en instituciones de salud, presentan desconfianza respecto a acudir a la consulta porque el médico los regaña, o porque el medicamento que dan en el sector público es de mala calidad. Todas las experiencias pueden ser ignoradas por la cultura dominante, minimizarlas, no comprenderlas o pasarlas desapercibidas.

En este apartado, también es común que las diferencias intergeneracionales (ya sea del personal de salud o del paciente) tengan un rol importante. Asimismo, el género, pues aun cuando la DM se presenta en ambos géneros, la prevalencia es más elevada en mujeres (aunque pudiera estar sesgada debido al subregistro en la población masculina), el rol que ha asumido la mujer sobre el cuidado de su enfermedad favorece la disposición de adoptar estilos de vida saludables, de acudir con mayor frecuencia a sus citas médicas o a los GAM (Cruz y cols., 2014). Asimismo, en este apartado, Kübler Ross propone identificar qué manifestaciones le permiten liberarse con mayor facilidad; es decir, una persona que se 
encuentra en la fase de ira puede resultarle más fácil expresar sus emociones según su edad y su género.

\section{Institucionalizar el conocimiento cultural.} El conocimiento sobre las fases de duelo y del bagaje cultural debería estar integrado a las intervenciones de salud. Por ello, es necesario que las intervenciones respondan a la diversidad cultural y que incluyan materiales didácticos sensibles a la comunidad. En este sentido, el equipo de salud debe ser capacitado para abordar e identificar las fases en las que se encuentra el paciente con DM, esto con el fin de lograr un adecuado manejo y aceptación del diagnóstico, así como el manejo de sus emociones y de conductas de alto riesgo, además de identificar las dificultades en el apego al tratamiento; de tal manera que logre el empoderamiento del paciente (Puente y cols., 1999). Estas unidades de salud presentan adecuaciones, según la cultura de la población, en sus diseños y espacios físico/arquitectónicos, en su mobiliario y mensajes orientadores (en lenguas o idiomas específicos si son requeridos por las características en la región). Además, en los procedimientos de atención se promueve una muy certera comunicación entre los usuarios y el personal para conocer sus expectativas respecto a la calidad del servicio en los procesos de atención (SSA, 2010).

5. Adaptarse a la diversidad. Los valores, conductas, actitudes, prácticas, políticas y estructuras que hacen posible la comunicación transcultural, conducen a una institución culturalmente competente. Cuando se reconocen, respetan y valoran todas las culturas y se integran esos valores en el sistema, las organizaciones culturalmente competentes pueden cumplir con las necesidades de grupos diversos. En este apartado, un estudio llevado a cabo por Brugada y colaboradores, muestra una intervención culturalmente competente con un paciente paquistaní con DM, quien utilizaba calzado inapropiado, y tenía creencias y prácticas musulmanas, y quien a través de esta cotidianidad presentó una lesión ulcerosa grado I (escala de Wagner) localizada en el dorso del pie izquierdo. Uno de los factores de riesgo que originó la úlcera, fue que el paciente manifestó orar sin protección sobre sus pies por prolongadas horas, lo cual el equipo médico desconocía, y sin una entrevista profunda, no se hubiera identificado. A partir de esto, se derivó a podología para el diseño de una ortesis de descarga de la zona afecta (Brugada y cols., 2013).

\section{Consideraciones finales}

La propuesta de integrar fundamentos de la psicología y tanatología para abordar al paciente con DM y preparar los Servicios de Salud desde la competencia cultural permite promover ambientes respetuosos, de servicio y de atención a los pacientes y el personal de salud (DGPLADES, 2014; Suh, 2014). Inicialmente es un llamado a las necesidades no satisfechas del paciente con DM, visualizar la propuesta teórica de Kübler-Ross y operacionalizarla en políticas públicas. La psicología no solo debe impactar en la clínica, sino posicionarse en las esferas de los tomadores de decisiones y proponer acciones locales con impacto global.

Por lo tanto, en este hueco del conocimiento y de la práctica cotidiana, la formación del profesional en psicología debe incluir estas herramientas y estar capacitado para intervenir. $\mathrm{Si}$ bien, no todas las estructuras curriculares incluyen la tanatología como una asignatura, es posible incorporar seminarios o talleres, de tal manera que adquiera este tipo de habilidades.

Asimismo, se hace una crítica al paradigma biologicista de los Servicios de Salud, pues a pesar que desde 1948 la OMS aseveró que la salud incluye elementos físicos, mentales y sociales, la inclusión de elementos psicológicos y sociales en los Servicios de Salud no han logrado afrontar las necesidades del paciente y su familia desde el contexto cultural. Por ello, 
la propuesta de abordaje culturalmente competente lleva una tendencia participativa que condensa una multitud de corrientes de pensamiento y acción. Destaca el propósito de promover y fortalecer la participación de los diversos grupos sociales en la autogestión de sus necesidades e intereses de corto, mediano y largo plazo. Esta propuesta se puede lograr conjuntamente con el médico o la enfermera, pues la salud es un bien preciado que no se valora hasta que se pierde. Desafortunadamente, las condiciones actuales impiden que exista una cultura hacia la salud; pero la propuesta de intervención tanatológica puede orientar el curso de vida del paciente con DM, y aunado a ello, un equipo de salud sensible a las necesidades socioculturales del paciente, puede crear una sinergia y lograr la adherencia terapéutica.
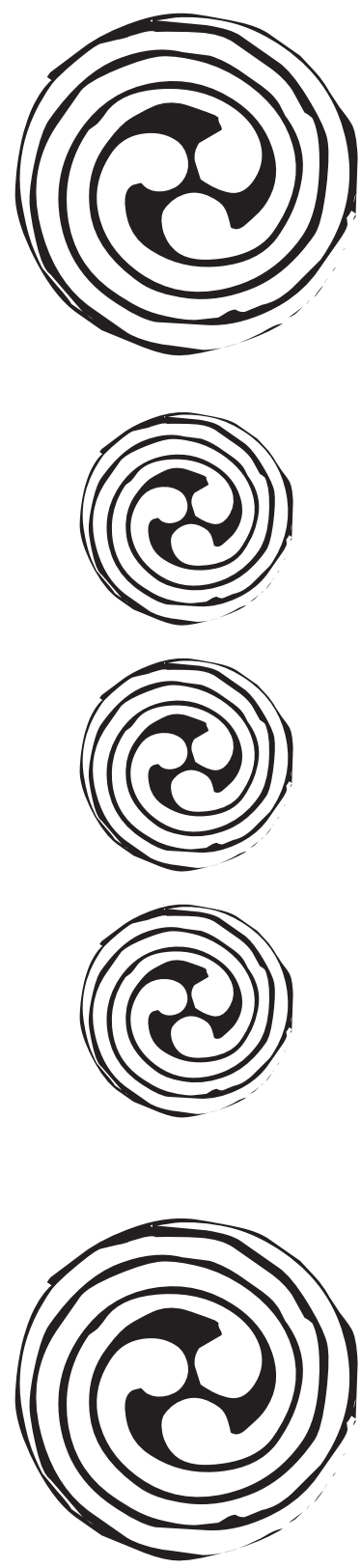


\section{Fuentes de consulta}

American Diabetes Association. (2011) Standards of medical care in diabetes 2011. Diabetes Care. Vol 34 Suppl 1 pp12-32.

Brugada Faura M, Medrano Jiménez R, Mora Morera O, Valverde Caballero I, Martín Zafra A. Salas ME. (2013) Diversity and universality in response to patient diabetic foot pakistani with type 2 diabetes mellitus from primarycare. Gerokomos. Vol 24, Núm 3, pp 139-143

Carrillo-Alarcón LC, López-López E, López-Carbajal MJ, Ortiz MI, Ponce-Montern HE, et al. (2015) Level of Knowledge in Patients with Type 2 Diabetes Mellitus and its Relationship with Glycemic Levels and Stages of Grief According to Kübler-Ross. J Diabetes Metab 6: 495. doi:10.4172/2155-6156.1000495.

Cruz-Bello P, Vizcarra-Bordi I, Kaufer-Horwitz M, Benítez-Arciniega AD, Ranjita Misra y ValdésRamos R. (2014) Género y autocuidado de la diabetes mellitus tipo 2 en el Estado de México. Papeles de población. Pap. Poblac. Vol 20, Núm 80, pp119-144.

Dirección General de Planeación y Desarrollo en Salud. (2014) Servicios y unidades de Salud, culturalmente Competentes. México Cosultado [25 de Seep 2014] disponible en [http://www.dgplades.salud.gob.mx/descargas/dmtdi/carpeta1/Serv_y_uni_Cult_Compt .pdf]

Dirección General de Epidemiología.(2015) Sistema de Vigilancia Epidemiológica Hospitalaria de Diabetes Tipo 2. Boletín de cierre anual 2015. Secretaría de Salud. Consultado [11 o c t u b e 2016$]$ D i s p o n i ble [http://www.epidemiologia.salud.gob.mx/doctos/infoepid/bol_diabetes/dm2_2015.pdf]

-(2016) Notificación Semanal Casos Nuevos de Enfermedades 2016. Información actualizada al mes de agosto de 2016. Consultado [11 octubre 2016] Disponible en [http://www.epidemiologia.salud.gob.mx/doctos/infoepid/inf_morbilidad/2016/INF_MO RBI_2016_08.pdf]

Dirección General de Promoción de la Salud. (2014) Estrategia nacional de promoción y prevención para una mejor salud. Mexico 2009. Consultado el día [13 Oct 2014] Disponible en [http://promocion.salud.gob.mx/dgps/descargas1/estrategia_nacional.pdf]

Federación Internacional de Diabetes.(2015) Atlas de la Diabetes de la FID. $7^{a}$ edición. Actualización de 2015. pp12-24

García R y Suárez R. (2007) La educación a personas con diabetes mellitus en la atención primaria de salud. Rev Cubana Endocrinol. Vol 18, Núm 1, pp1-13

Grossman D. (1994) Enhancing your 'cultural competence'. American Journal of Nursing. Vol 94, Núm 7, pp 58-62.

Jodelet D. (1993) La representación social: fenómenos, concepto y teoría. In: Moscovici S, editor. Psicología social II. Barcelona: Paidos. p. 469-94

Kleiman A. Benson P. (2006) Anthropology in the Clinic: The Problem of Cultural Competence and How to Fix It. Plos Medicine. Vol3, Núm. 10 pp 1673-1676.

Kübler Ross E. (1969) Sobre la muerte y los moribundos. Grijalbo. México.
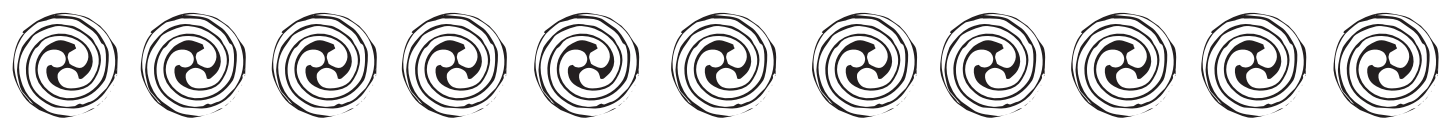

๑ $80 @$ 
Lara Esqueda A, Aroch Calderón A, Jiménez RA, Arceo Guzmán M, Velázquez Monroy O (2004). Grupos de Ayuda Mutua: Estrategia para el control de diabetes e hipertensión arterial. Archivos de Cardiología de México. Vol 74 Núm 4, pp330-336.

López-López E, Gutiérrez-Soria D, Idrovo AJ. (2012) Evaluation of a diabetes care program using the effective coverage framework Int J Qual Health Care. Vol 24, Núm 6, pp 619-25.

Moxley A, Mahendra N, \& Vega-Barachowitz C. (2004) Cultural competence in health care. The ASHA Leader. Vol 13 pp 6-22

Organización Mundial de la Salud. (2000) iActuemos ya! En contra de la Diabetes. Suiza.

- (1991) Directrices para el desarrollo de un programa nacional para la diabetes mellitus. Ginebra: OMS. División de enfermedades no transmisibles y tecnología de la salud; informe OMS/DBO/DM91-1

Puente González G, Salinas Martínez AM, Villarreal Ríos E, Albarrán Gómez T, Contreras Patiño T, Elizondo García R. (1999) Costo Costo-efectividad efectividad de un programa educativo para de un programa educativo para diabéticos tipo 2. Rev Enferm IMSS; Vol 7, Núm 3, pp147-150.

Rodríguez-Moctezuma, José Raymundo; López-Delgado, María Elisa; Ortiz-Aguirre, Alma Rosa; Jiménez-Luna, Jorge; López-Ocaña, Luis Rafael; Chacón-Sánchez, Jesús. (2015) Etapas del duelo en diabetes y control metabólico. Revista Médica del Instituto Mexicano del Seguro Social. Vol 53, Núm 5, pp 546-551

Salleras-San Martín L.(2005) Educación Sanitaria. Madrid: Díaz de Santos, pp 20-26

SSA (1998) Coordinación de Vigilancia Epidemiológica. Programa de Salud del adulto y el Anciano. Guía Técnica para el Funcionamiento de Clubes de Diabéticos. México.

- (2010) Norma Oficial Mexicana NOM-015-SSA2-2010, Para la prevención, tratamiento y control de la diabetes mellitus. México

- (2001) Guía técnica para capacitar al paciente con DM. México.

Solís Lizama M, Fortuny Loret de Mola P. (2010) Otomíes hidalguenses y mayas yucatecos. Nuevas caras de la migración indígena y viejas formas de organización. Migraciones internacionales. Vol 5, Núm 4, pp 101-138.

Suh E. (2004) The Model of Cultural Competence Through an Evolutionary Concept Analysis. Journal of Transcultural Nursing. Vol 15, Núm 2, pp 93-102.

The International Expert Committee. (2009) International Expert Committee Report on the Role of the A1c Assay in the Diagnosis of Diabetes.Diabetes Care Vol.32.pp1327-1334.

Torres López TM, Sandoval-Díaz M, Pando-Moreno M. (2005) Sangre y azúcar: representaciones sobre la diabetes de los enfermos crónicos en un barrio de Guadalajara, México. Cad. Saúde Pública, Rio de Janeiro. Vol21, Núm 1, pp101-110

Wilches-Gutiérrez JL, Arenas-Monreal L, Paulo-Maya A, Peláez-Ballestas I, Idrovo AJ (2012). A 'beautiful death': mortality, death, and holidays in a Mexican municipality. Soc Sci Med. Vol 74,Núm 5, pp 775-82 
